\title{
NOTE \\ SUR LA PISCICULTURE ARTIFICIELlE DU SANDRE
}

\author{
par P. CHIMITS \\ Ingénieur des Eaux et Forêts.
}

Dans le numéro de Juillet-Septembre 1948 du Bulletin français de Pisciculture, nous avons rendu compte de notre mission piscicole en Tchécoslovaquie, au cours de laquelle nous avons visité diverses piscicultures et ramené par avion des reproducteurs de Carpes de la race de Wittingau, ainsi que des œufs embryonnés de Sandre.

Le Sandre est rare en France, puisqu'on ne le trouve que dans les affluents du Rhin, dans la Saône et dans le Rhône. Sa chair est particulièrement estimée dans toute l'Europe centrale; de fait, nous avons eu l'occasion d'en goûter au cours de notre mission et l'avons trouvée excellente, comparable à celle de la grosse Perche et peut-être mème plus fine. Ce poisson commence à être apprécié des Parisiens car les halles, à certaines périodes, en reçoivent de Hollande.

Les pêcheurs sportifs ont eu leur attention attirée par la belle taille de ce poisson carnassier. Il y a actuellement un engouement de certaines sociétés de pêche pour le repeuplement en Sandres de leurs eaux; nous avons même pu nous rendre compte, au cours d'un voyage récent au Maroc, que le Service forestier en avait commencé l'introduction.

Nous croyons donc utile d'indiquer ci-dessous la technique spéciale de la reproduction artificielle du Sandre telle que nous l'avons vu pratiquer en 1948 au domaine piscicole d'État de Trébon (ex-Wittingau), en Tchécoslovaquie.

Le Sandre pond à la fin du printemps, normalement fin Avril et courant Mai quand l'eau atteint $15^{\circ}$ environ. C'est aux environs du

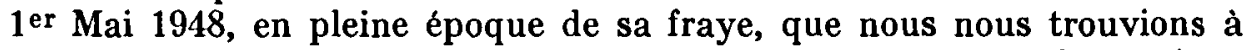
Wittingau. Il est probable qu'en France, et surtout dans les régions méridionales, sa ponte aurait lieu courant Avril, c'est-à-dire après celle de la Perche et avant celles de la Tanche et du Gardon.

\section{Bassin de ponte ot ponte.}

La reproduction artificielle du Sandre ne ressemble en rien à la fécondation des cufs de Salmonides et de Brochets : elle se rapproche plutôt de celle de la Carpe en frayère Dubisch.

A Trébon (ex-Wittingau), les bassins utilisés pour cette ponte sont les bassins de stockage et d'hivernage des Carpes et Tanches produites 
par les 6.000 hectares d'étangs du domaine. La vente de ces poissons étant échelonnée pendant tout l'hiver et se terminant début Avril, ces bassins peuvent dès lors être utilisés à d'autres fins. Ils ont 20 mètres de long sur 10 de large, une profondeur de $1 \mathrm{~m}$. 50 , et sont à bords perrés et à fond de sable. Chaque bassin est alimenté par un débit de 2 à 5 litresseconde, le jet étant brisé sur une planche pour oxygéner l'eau au maximum. Il semble que l'on puisse utiliser des surfaces d'eau plus réduites.

Le bassin est mis à sec pendant quelques jours, puis rempli de 50 à 80 centimètres d'eau. Quand cette eau atteint 14 ou $15^{\circ}$, on introduit 3 ou 4 couples de géniteurs pesant de 1 à $3 \mathrm{kgs}$. On peut, dans un coin du bassin, placer quelques planches sur des piquets, au ras de l'eau, pour constituer un abri à ces géniteurs.

Le Sandre ne pondant que sur des végétaux immergés, il ne peut lâcher ses œufs sur le fond de sable ; aussi met-on sur le fond du bassin 4 ou 5 disques de 50 centimètres de diamètre environ, hauts de 8 à 10 centimètres et constitués d'une sorte de feutrage de filaments serrés et blanchâtres (nous en indiquons plus loin la nature). Ces disques sont fixés sur le fond de sable par des piquets en bois. Il n'y a plus alors, comme pour les frayères Dubisch, qu'à laisser faire la nature et à attendre que mâles et femelles viennent se livrer à leurs ébats amoureux sur les disques. De fait, si la température est convenable et les reproducteurs en bon état, on peut voir, dans un délai de trois ou quatre jours, des quantités d'œufs déposés sur les filaments des disques. Ces œufs sont de petites sphères translucides de 1 millimètre à $1 \mathrm{~mm}$. 5 de diamètre; on estime qu'une femelle en pond 150 à 200.000 par $\mathrm{kg}$. de son poids; mais il se peut que pour des causes défavorables (notamment un abaissement brutal de la température) la ponte n'ait pas eu lieu; on remplacera alors les disques par d'autres, car leur surface se sera probablement couverte d'un enduit gluant sur lequel les géniteurs se refuseront à pondre; ces disques pourront, d'ailleurs, resservir après avoir été nettoyés et séchés.

\section{Récolte et transport des cufs embryonnés.}

L'incubation est plus longue que pour les œufs de Carpe, qui n'exigent pour éclore que quatre à cinq jours, et dure à peu près comme celle des œufs de Brochet, soit dix jours environ. Dès le septième jours après la ponte, les œufs peuvent être transportés. Le disque est alors enlevé avec précaution, fractionné en morceaux que l'on place dans des caisses de transport semblables à celles utilisées pour les ceufs de Truite ou de Brochet, mais, évidemment, sans emploi de clayettes. La caisse est calorifugée et les couches de disques sont surmontées d'une caissette à glace fondante; tout comme pour le Brochet, le transport des oufs peut durer trente-six heures. Il est imprudent de tenter des délais plus longs.

\section{Mise en place des œuf́ embryonnés.}

On se gardera bien de détacher les cufs de l'entrelacement des filaments du disque. On placera simplement les fragments de disque supportant 

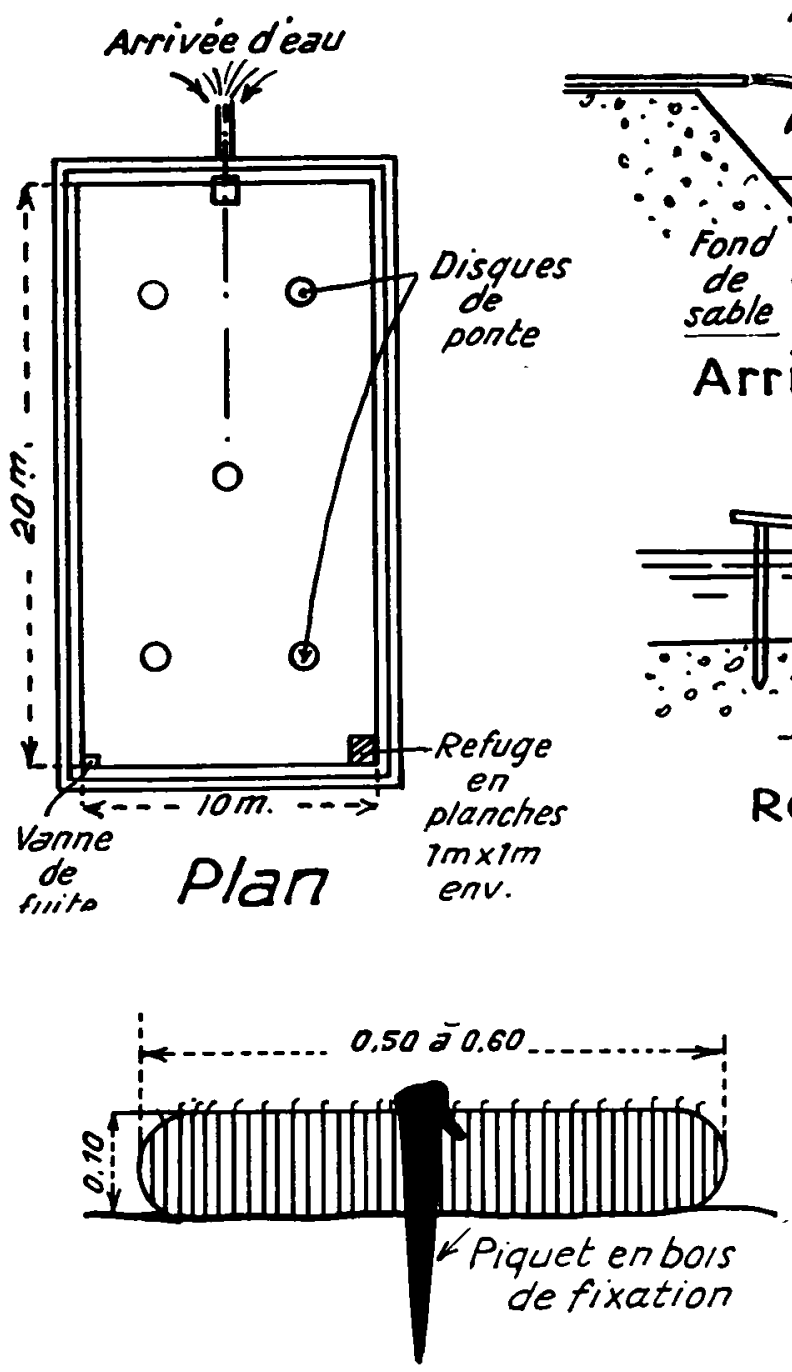

Planchette $0,30 \times 0,30$

sur piquet pour
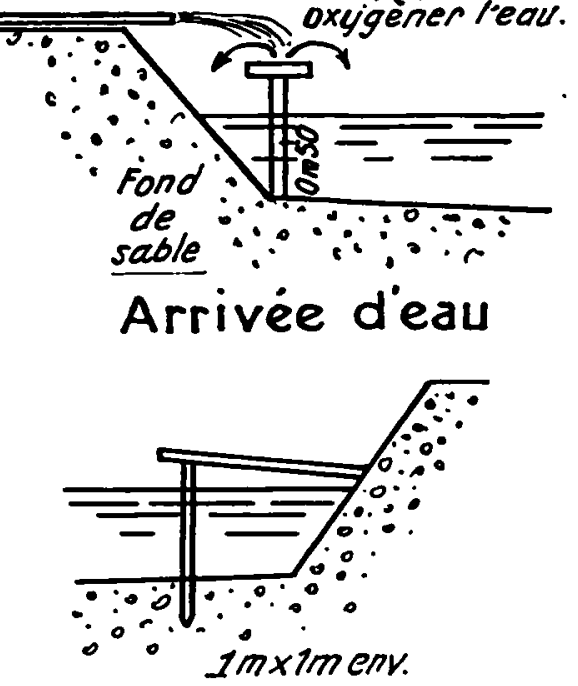

Refuge
Disque de ponte en racines de carex

Cloche d'immersion en osier pour oeufs embryonnés de Sandre

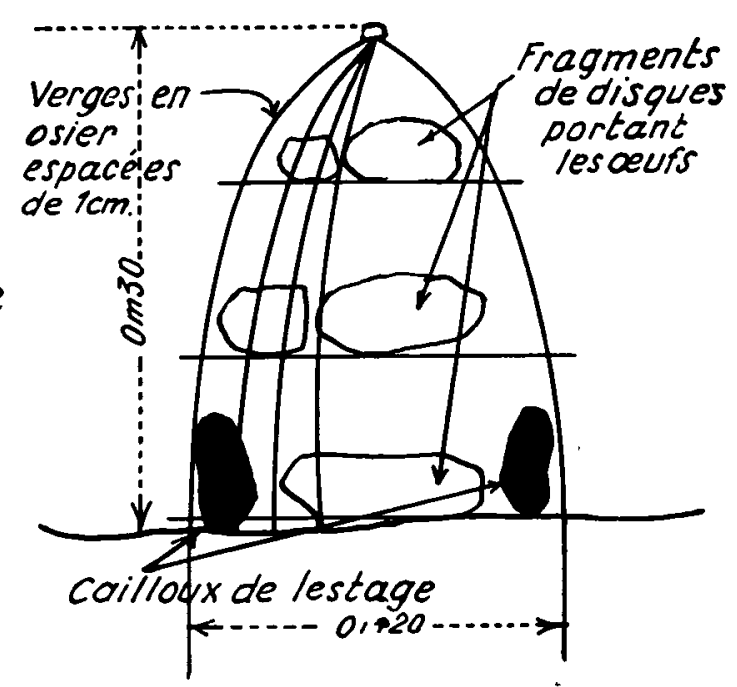

BASSIN DE PONTE POUR LE SANDRE 
les œufs dans la rivière ou la pièce d'eau à aleviner en les fixant au fond avec des piquets de bois. Naturellement, on choisira une eau tranquille, sans courant, s'il s'agit d'une rivière, mais surtout à fond de sable propre : le Sandre, et surtout ses œufs, craignent en effet la vase.

Les tchèques utilisent, pour contenir les fragments de disque, de curieux petits paniers en osier en forme de cloche, hauts de 30 centimètres et larges de 20 , dont l'écartement des verges est de 1 centimètre environ : ainsi les cufs sont à l'abri des poissons toujours amateurs de la fraye des autres, et les petits Sandres, une fois éclos, s'y réfugient (paraît-il) à la moindre alerte. Le panier est lesté de cailloux ou fixé au fond par un piquet.

\section{Nature des disques de ponte.}

Nous avons évidemment tenu à connaître la nature du feutrage de filaments blanchâtres constituant les disques de ponte; il fut assez difficile de nous le faire expliquer : il s'agit tout simplement de touffes de racines de carex que l'on trouve dans les queues et bords d'étangs et de marais siliceux et qu'on appelle tourbettes ou touradons. Ces touffes, constituées d'un entrelacement très serré et blanchâtre de racines étroitement mêlées, sont tranchées à l'aide d'une sorte de faulx et soigneusement lavées pour en sortir le dépôt de vase et d'humus.

\section{Conclusion.}

Nous espérons que cette note facilitera en France la propagation du Sandre, sur lequel nous estimons utile de faire les observations suivantes:

- au point de vue culinaire, c'est un excellent poisson d'eau douce, supérieur à la Perche et au Black-bass;

- au point de vue piscicole, c'est un carnassier; il exige beaucoup de blanchaille et se capture au vif et avec les engins artificiels du lancer léger (cuillères, devons) ;

- au point de vue sportif, s'il fait honneur au pêcheur qui l'a capturé de par son poids qui est respectable, puisqu'il atteint 5 à $6 \mathrm{kgs}$, sa défense est médiocre, inférieure à celle du Brochet et surtout du Black-bass; après quelques violents coups de queue, il se "rend " assez vite.

Au point de vue écologique, il préfère les étangs et les grandes rivières comportant, au moins en certains endroits, des fonds sableux pouvant lui servir de frayère. Il exige naturellement des eaux riches en fretin, car il est très vorace. Il tolère les eaux froides; aussi, à notre avis, est-il plus à sa place dans l'Est de la France que dans l'Ouest et le Sud-Ouest, où le Black-bass, moins grand mais plus sportif, doit, à notre avis, lui être préféré. 